DOI: $10.12737 /$ article_59c22a09309069.72810475

УДК 621.833

ПРИМЕНЕНИЕ «УСЛОВИЯ СТЕФАНА» ДЛЯ РЕШЕНИЯ ТЕПЛОВЫХ ЗАДАЧ В ОБЪЕКТАХ ЛЕСНОГО КОМПЛЕКСА

кандидат физико-математических наук, доцент Б. М. Кумицкий ${ }^{1}$

кандидат технических наук С. В. Малюков ${ }^{2}$

кандидат физико-математических наук Н. А. Саврасова ${ }^{3}$

кандидат технических наук С. В. Чуйкин ${ }^{1}$

1 - ФГБОУ ВО «Воронежский государственный технический университет», г. Воронеж, Российская Федерация 2 - ФГБОУ ВО «Воронежский государственный лесотехнический университет им. Г.Ф. Морозова»,

г. Воронеж, Российская Федерация

3 - Военный учебно-научный центр Военно-воздушных сил «Военно-воздушная академия имени профессора

Н.Е. Жуковского и Ю.А. Гагарина», г. Воронеж, Российская Федерация

Рассмотрена двухфазная постановка задачи о «тепловом ударе» при соприкосновении в начальный момент времени двух однородных полупространств с разными фазами и температурами, отличными от температуры фазового перехода. В отсутствие конвекции и тепловых источников для постоянных теплофизических параметров такая задача может быть сформулирована как задача о сопряженности двух температурных полей на движущемся фронте затвердевания с дополнительными граничными условиями (условия Стефана). С практической точки зрения такого рода задачи могут применяться при исследовании процессов, протекающих в объектах лесного комплекса: при производстве древесностружечных плит, устройстве прудов и водоемов, промерзании (оттаивании) грунтов и т. д. Решение проводилось с использованием интегрального преобразования Лапласа. Полученная при этом точная аналитическая зависимость в явном виде определяет закон движения межфазной границы и полностью отражает картину распределения температур в обеих фазах. Этим показана эффективность применения метода интегрального преобразования Лапласа для решения тепловых и диффузионных задач, в том числе и пространственных для различных краевых условий. Полученное поле температур соответствует известному распределению Гаусса, а скорость движения межфазной границы обратно пропорциональна корню квадратному времени кристаллизации. Данные примерного числового расчета, проведенного для системы вода - лед, соответствуют скорости промерзания (таяния) приблизительно $10^{-3} \mathrm{Mm} / \mathrm{c}$. Полученные результаты могут быть использованы при исследовательских работах в области строительной теплофизики, геофизики и металлургии.

Ключевые слова: условие Стефана, преобразование Лапласа, водоем, оригинал.

\title{
APPLICATION OF «STEFAN CONDITIONS» FOR SOLVING HEAT TASKS IN OBJECTS OF THE FOR- EST COMPLEX
}

Candidate of Physical and Mathematical Sciences, Associate Professor B. M. Kumitsky ${ }^{1}$

Candidate of Technical Sciences S. V. Malyukov ${ }^{2}$

Candidate of Physical and Mathematical Sciences N. A. Savrasova ${ }^{3}$

Candidate of Technical Sciences S. V. Chuikin ${ }^{1}$

1 - Federal State Budget Education Institution of Higher Education «Voronezh State Technical University», Voronezh, Russian Federation

2 - Federal State Budget Education Institution of Higher Education «Voronezh State University of Forestry and Technologies named after G.F. Morozov», Voronezh, Russian Federation

3 - The military educational and scientific center of the Air Force «The Air Force Academy named after Professor N.E. Zhukovsky and Yu.A. Gagarin», Voronezh, Russian Federation 


\begin{abstract}
A two-phase formulation of the problems of "thermal shock" is considered when two homogeneous half-spaces come into contact at an initial instant of time with different phases and temperatures different from the phase transition temperature. In the absence of convection and thermal sources for constant thermophysical parameters, which can be formulated as the problem of conjugation of two temperature fields on the moving solidification front with additional boundary conditions (Stefan conditions). From a practical point of view, this kind of problem can be the arrival of processes occurring in the objects of the forest complex: in the production of particle board, the processing of ponds and reservoirs, the freezing (thawing) of soils, etc. The solution was carried out using the Laplace integral transformation. The exact analytical dependence obtained in this way explicitly determines the law of interference in each phase. These functions are used for integral transformations. The resulting temperature field corresponds to the known Gaussian distribution, and the velocity of the interphase boundary movement is inversely proportional to the square root of the crystallization time. The data of the approximate numerical calculation carried out for the water-ice system corresponds to a freezing (thawing) rate of approximately $10-3 \mathrm{~mm} / \mathrm{s}$. The obtained results can be used for research work in the field of construction thermal physics, geophysics and metallurgy.
\end{abstract}

Keywords: Stefan's condition, Laplace transform, reservoir, original

Явления фазовых переходов, когда вещество переходит из одного агрегатного состояния в другое с поглощением или выделением тепла, наблюдаются в различных природных и технологических процессах. Поэтому возникает необходимость в описании этих явлений. Подобного рода задачи имеют большое практическое значение в металлургии [1], строительной теплофизике, промерзании и оттаивании грунтов под строениями [2, 3, 4], производстве льда [5], теплотехнике [6,7] и других прикладных дисциплинах.

Изменение агрегатного состояния, в общем случае, сопровождается фазовым превращением первого рода с выделением скрытой теплоты кристаллизации (плавления) при неизменной температуре на границе раздела фаз, а также скачкообразным изменением некоторых теплофизических свойств вещества, влияющих на протекание тепловых процессов. Кроме того, происходит движение межфазной границы по заранее неизвестному закону.

Особенность таких задач заключается в том, что возникает необходимость решать два уравнения теплопроводности (отдельно для каждой фазы) при дополнительных краевых условиях и сопряженности температурных полей на движущемся фронте межфазной границы, что приводит к нелинейности решения.

Задачи данного класса с подвижными межфазными границами получили название задач Стефана, а уравнение теплового баланса на фронте кристаллизации с учетом скрытой теплоты и сопряжения фаз называют условиями Стефана $[8,9$, $10,11]$.

При решении задач Стефана с различными краевыми условиями, наряду с классическими методами (метод разделения переменных, метод источников и др.) [3], используются численные методы $[4,11]$. Но классические методы не всегда эффективны, а полученные решения не всегда удобны для практического применения. Более перспективным является использование методов интегральных преобразований, например, Фурье [12].

Более широкое использование в решении тепловых задач получил метод преобразования Лапласа $[7,13]$, позволяющий: сводить сложные задачи к более простым; получить конечный результат в явном виде; решать пространственные задачи; считать решение простых задач совершенно строгим.

Основной предпосылкой при моделировании фазовых превращений системы жидкость - твердое тело является тот факт, что фазовый переход совершается при заданной постоянной температуpe $\mathrm{T}_{\Phi}$.

Рассмотрим одномерную двухфазную тепловую задачу в классической постановке Стефана, известную в литературе как «вторая задача Стефана» $[9,14,15,16,17,18]$. Пусть фазовый переход происходит на подвижной границе раздела фаз $x=S(t)$, которая делит бесконечное пространство $\Omega$, заполненное теплопроводящей средой, на две 
полуограниченные области $\Omega_{1}(t)$ и $\Omega_{2}(t)$, занятые соответственно твердой и жидкой фазами. Температуры фаз в начальный момент времени равны, соответственно, $T_{1}(x, 0)=T^{*}<T_{S}, T_{2}(x, 0)=T_{0}>$ $T_{S}$ (рис. 1).

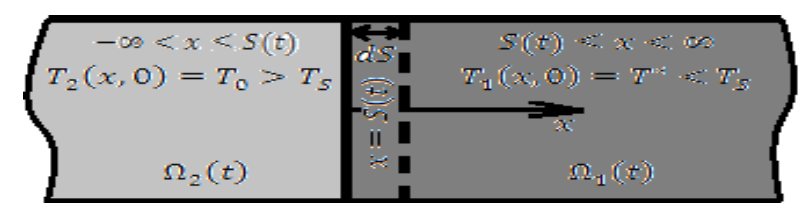

Рис. 1. Геометрическая схема тепловой задачи о движении межфазной границы

Итак, в начальный момент времени $t=0$ происходит соприкосновение областей $\Omega_{1}(t)$ и $\Omega_{2}(t)$ в форме «теплового удара» [9], и на поверхности соприкосновения скачком устанавливается, а затем поддерживается температура фазового перехода $T_{S}$, для которой справедливо неравенство $T^{*}<T_{S}<T_{0}$. В результате этого образуется слой $d S$, толщина которого со временем увеличивается.

Требуется найти закон движения фронта кристаллизации и распределение температур в твердой и жидкой фазах, т. е. решить задачу о сопряжении двух температурных полей на движущемся фронте кристаллизации. Для нахождения поля температур необходимо решить систему двух уравнений теплопроводности, которые, в отсутствие конвекции и теплоисточников для постоянных теплофизических характеристик, имеют вид соответственно для твердой и жидкой фаз

$$
\frac{\partial T_{1}}{\partial t}=a_{1} \frac{\partial^{2} T_{1}}{\partial x^{2}}
$$

для $\Omega_{1}(t) \quad(S(t) \leq x<\infty)$,

$$
\frac{\partial T_{2}}{\partial t}=a_{2} \frac{\partial^{2} T_{2}}{\partial x^{2}}
$$

для $\Omega_{2}(t) \quad(-\infty<x \leq S(t))$.

Так как межфазная граница движется по неизвестному закону, то на ней, кроме граничных условий для уравнений (1) и (2) $T_{1}[S(t), t]=$ $T_{2}[S(t), t]=T_{S}$ должно быть еще одно условие (условие Стефана), определяющее скорость движения границы

$$
\left.\lambda_{1} \frac{\partial T_{1}}{\partial x}\right|_{x=S}-\left.\lambda_{2} \frac{\partial T_{2}}{\partial x}\right|_{x=S}=-L \rho_{1} \frac{d S}{d t} .
$$

Здесь $a_{1}, \lambda_{1}, a_{2}, \lambda_{2}$ - коэффициенты температуропроводности и теплопроводности для твердой и жидкой фаз соответственно;

$L$ - скрытая теплота плавления;

$\rho_{1}-$ плотность твердой среды;

$\frac{d s}{d t}-$ скорость движения межфазной границы.

Отрицательная правая часть выражения (3) указывает на процесс плавления твердой фазы, для которого справедливо $\frac{d s}{d t}<0$.

Для решения уравнений теплопроводности (1) и (2) используем операционный метод преобразования Лапласа $[7,13]$, который состоит в том, что решение ищется не для самой функции времени $f(t)$, а для ее изображения $\bar{f}(P)$. Переход к изображению осуществляется при помощи преобразования относительно временной переменной $t$

$$
\bar{f}(P)=\int_{0}^{\infty} f(t) e^{-P t} d t,
$$

где $P$ - комплексное число (параметр Лапласа).

Когда найдено решение уравнения в изображениях, остается определить его оригинал с помощью обратного преобразования Лапласа по формуле обращения

$$
f(t)=\frac{1}{2 \pi i} \int_{\sigma-i \infty}^{\sigma+i \infty} \bar{f}(P) e^{P t} d P .
$$

Контурное интегрирование (5) осуществляется методом функций комплексного переменного [19].

В большинстве же случаев обратное преобразование может быть выполнено на основе таблиц стандартных изображений.

Применим преобразование Лапласа (4) к уравнениям (1) и (2), что равносильно умножению их правой и левой частей на $e^{-P t}$ с последующим интегрированием по tот 0 до $\infty$ :

$$
\int_{0}^{\infty} e^{-P t} \frac{\partial T_{1}}{\partial t} d t=a_{1} \int_{0}^{\infty} e^{-P t} \frac{\partial^{2} T_{1}}{\partial x^{2}} d t .
$$

Интегрируя по частям левую часть (6), получим

$$
\begin{aligned}
\int_{0}^{\infty} e^{-P t} \frac{\partial T_{1}}{\partial t} d t= & e^{-P t} T_{1}(x, t) \mid{ }_{0}^{\infty} \\
& +P \int_{0}^{\infty} e^{-P t} T_{1}(x, t) d t \\
& =-T_{1}(x, 0)+P \bar{T}_{1}(x, P)- \\
& =P \bar{T}_{1}(x, P)-T^{*}
\end{aligned}
$$

Правая часть выражения (6) примет вид: $a_{1} \frac{d^{2} \overline{T_{1}}}{d x^{2}}$. Приравнивая после преобразования правую 


\section{Естественные науки и лес}

и левую части (6), проводя аналогичные операции с уравнением (2), получим соответствующие изображающие уравнения:

$$
\begin{aligned}
& P \overline{T_{1}}(x, P)-T^{*}=a_{1} \frac{d^{2} \overline{T_{1}}(x, P)}{d x^{2}} ; \\
& P \overline{T_{2}}(x, P)-T_{0}=a_{2} \frac{d^{2} \overline{T_{2}}(x, P)}{d x^{2}} .
\end{aligned}
$$

Они являются обыкновенными линейными, неоднородными уравнениями второго порядка с постоянными коэффициентами. Их решения в пространстве изображений находятся в виде суммы решений общего однородного и частного неоднородного уравнений [19]. В случае равномерного начального распределения температур частные решения уравнений (7) и (8) являются очевидными, так как $\frac{d^{2} \bar{T}}{d x^{2}}=0$ :

$$
\begin{aligned}
& {\overline{T_{1}}}_{\text {YAC }}=\frac{T^{*}}{P} ; \\
& {\overline{T_{2}}}_{\text {YAC }}=\frac{T_{0}}{P} .
\end{aligned}
$$

Для нахождения общих решений ${\overline{T_{1}}}_{O Б}$ и $\bar{T}_{2}$ ОБ составляются характеристические алгебраические уравнения (в данном случае квадратные). На основании замены $\frac{d^{2} \bar{T}}{d x^{2}}=\theta^{2}$ получаем $a \theta^{2}=P$, откуда $\theta_{1,2}= \pm \sqrt{\frac{P}{a}}$, а соответствующие решения (7) и примут вид

$$
\begin{aligned}
& T_{1}(x, P)=A e^{\sqrt{\frac{P}{a_{1}}} x}+B e^{-\sqrt{\frac{P}{a_{1}}} x}+\frac{T^{*}}{P}, \\
& T_{2}(x, P)=C e^{\sqrt{\frac{P}{a_{2}}} x}+D e^{-\sqrt{\frac{P}{a_{2}}} x}+\frac{T_{0}}{P} .
\end{aligned}
$$

Здесь $A, B, C, D$ - произвольные постоянные, которые однозначно определяются из граничных условий. Для этого с помощью преобразования Лапласа их необходимо перевести в пространство изображений:

$$
\begin{aligned}
& T_{1} \mid x=S(t)=\int_{0}^{\infty} e^{-P t} T_{S} d t=\frac{T_{S}}{P} \\
& T_{2} \mid x=S(t)=\int_{0}^{\infty} e^{-P t} T_{S} d t=\frac{T_{S}}{P}
\end{aligned}
$$

При $x \rightarrow \infty$ слагаемое $A e^{\sqrt{\frac{P}{a_{1}} x}}$ нефизично, поэтому следует положить $A=0$, постоянную $B$ найдем из условия на границе $x=S(t)$ с учетом (13). Тогда

$$
B=\frac{T_{S}-T^{*}}{P} e^{\sqrt{\frac{P}{a_{1}}} S}
$$

Поступая аналогично с выражением (12) с учетом (14), получим значения для постоянных $D$ и $C$ :

$$
D=0 C=\frac{T_{S}-T_{0}}{P} e^{-\sqrt{\frac{P}{a_{2}}} S} .
$$

Изображающие уравнения (11) и (12) теперь примут вид

$$
\begin{aligned}
& \overline{T_{1}}(x, P)=\frac{T_{S}-T^{*}}{P} e^{\sqrt{\frac{P}{a_{1}}}(S-x)}+\frac{T^{*}}{P} ; \\
& \bar{T}_{2}(x, P)=\frac{T_{S}-T_{0}}{P} e^{\sqrt{\frac{P}{a_{2}}}(x-S)}+\frac{T_{0}}{P} .
\end{aligned}
$$

По таблице обратных преобразований Лапласа находим оригинал распределения температур (17) и (18) [21, табл. 8.4-1, с. 227, 231]:

$$
\begin{aligned}
& \overline{T_{1}}(x, t)=\left(T^{*}-T_{S}\right) \operatorname{erf}\left(\frac{x-S}{2 \sqrt{a_{1} t}}\right)+T_{S}, \\
& \bar{T}_{2}(x, t)=\left(T_{0}-T_{S}\right) \operatorname{erf}\left(\frac{S-x}{2 \sqrt{a_{2} t}}\right)+T_{S} .
\end{aligned}
$$

Видно, что поле температур (19) и (20) подчиняется известному распределению ЛапласаГаусса. Входящие в эти выражения функции $\operatorname{erf}\left(\frac{x-s}{2 \sqrt{a_{1} t}}\right)$ и $\operatorname{erf}\left(\frac{S-x}{2 \sqrt{a_{2} t}}\right)$ представляют собой соответствующие интегралы вероятностей, обладающие свойствами:

$$
\begin{aligned}
\operatorname{erf}(z)=\frac{2}{\sqrt{\pi}} \int_{0}^{\infty} e^{-z^{2}} d z & \\
=\frac{2}{\sqrt{\pi}}\left(z-\frac{z^{3}}{3}+\frac{z^{5}}{10}-\frac{z^{7}}{42} \pm \cdots\right) & \\
\operatorname{erf}(0)=0 ; \quad \operatorname{erf}(\infty)=1 ; & \operatorname{erf}(-z)=-\operatorname{erf}(z) .
\end{aligned}
$$

Для выполнения условия Стефана продифференцируем по $х$ уравнения (19) и (20), а полученные выражения подставим в (3).

$$
\lambda_{1} \frac{T^{*}-T_{S}}{\sqrt{\pi a_{1} t}}-\lambda_{2} \frac{T_{0}-T_{S}}{\sqrt{\pi a_{2} t}}=-L \rho_{1} \frac{d S}{d t}
$$

или, учитывая связь $a_{i}=\frac{\lambda_{i}}{c_{i} \rho_{i}}(i=1,2)$ и введя обозначение

$$
\beta=\frac{\left(T_{0}-T_{S}\right) \sqrt{\lambda_{2} \rho_{2} c_{2}}-\left(T^{*}-T_{S}\right) \sqrt{\lambda_{1} \rho_{1} c_{1}}}{L \rho_{1} \sqrt{\pi}},
$$

получим

$$
\frac{d S}{d t}=\frac{\beta}{\sqrt{t}} .
$$

Результат для скорости движения межфазной границы (23) качественно соответствует полученному иным методом в работе [22], а также автомодельным решениям [8, 12], в которых движение фронта промерзания по такому закону, по существу, является аксиомой. 
Интегрируя (22), получим величину роста приграничного слоя

$$
S=2 \beta \sqrt{t}+K .
$$

Из предположения, что $S=0$ для момента времени $t=0$, следует, что $K=0$. Тогда окончательно

$$
S=2 \beta \sqrt{t} .
$$

Если значение $\sqrt{t}$ подставить в формулу для распределения температур в твердой и жидкой фазах (19), (20), то можно получить стационарную температурную зависимость, графически представленную на рис. 2.

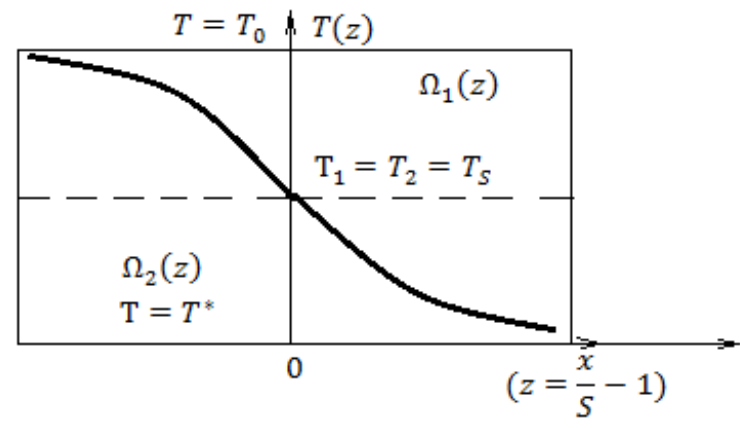

Рис. 2. Установившееся распределение температур в среде, заполняющей неограниченное пространство $\Omega=\Omega_{1}+\Omega_{2}$, включающее в себя твердую $\Omega_{1}$ и жидкую $\Omega_{2}$ фазы

В качестве сравнения приведем примерный расчет для системы вода - лед по формулам (23), (24) для температуры фазового перехода $T_{S}=273 \mathrm{~K}$, начальных температур твердой и жидкой фаз соответственно $T^{*}=263 \mathrm{~K}, T_{0}=300 \mathrm{~K}$ при следующих значениях теплофизических параметров: $L=333700$ Дюс/кг, $\rho_{1}=900 \kappa 2 / \mathrm{m}^{3}, \rho_{2}=1000 \kappa 2 / \mathrm{m}^{3}, c_{1}=2000$ Дже/(каК), $c_{2}=1200$ Дж/(кгК), $\lambda_{1}=2,23$ Дж/кг, $\lambda_{2}=0,58$ Дж $/ \kappa 2$.
Подставляя эти данные в (23) и (24), получим

$$
S=334 \cdot 10^{-6} \sqrt{t} .
$$

Оценочный результат, полученный по формуле (25), дает суточный прирост толщины льда около $10 \mathrm{~cm}$, что соответствует скорости промерзания порядка $10^{-3} \mathrm{Mm} / \mathrm{c}$.

Проделанная работа позволяет сделать следующие выводы:

1. Показана эффективность применения метода интегральных преобразований Лапласа для решения нестационарных задач теплопроводности и диффузии, в частности, при выборе теоретического режима производства древесностружечных плит [16].

2. Получены точные аналитические решения второй задачи Стефана в одномерной постановке.

3. Распределение температур и закон движения межфазной границы качественно соответствуют полученным ранее с помощью автомодельных решений и численных методов.

4. Проведенные оценочные расчеты для системы вода - лед дают разумные результаты для скорости движения границы промерзания.

5. Полученное уравнение движения межфазной границы может быть использовано для описания перемещения фронта парообразования при производстве фанеры и плиточных материалов отходов древесины.

\section{Библиографический список}

1. Чубинский, А.Н. Моделирование процессов склеивания древесных материалов [Текст] / А.Н. Чубинский, В.В. Сергеевичев. - СПб.: дом Герда, 2007. - 176 с.

2. Кумицкий, Б.М. Тепловые процессы при остывании водного бассейна [Текст] / Б.М. Кумицкий, Н.А. Саврасова, С.В. Чуйкин // Международная научно-практическая конференция «Развитие идей Г.Ф. Морозова при переходе к устойчивому лесоуправлению» 20-21 апреля 2017 г. Сб. науч. ст. по материалам.- Воронеж: ВГЛТУ, 2016. - С. 208-212.

3. Бартенев, И.М. Экологизация технологии и лесной техники [Текст] / И.М. Бартенев, В.Н. Винокуров // Лесное хозяйство. - 199. - № 4-5. - С. 5-7.

4. Матвеев, Н.Н. Аномалии тепловых свойств целлюлозы при переходах кристалл-кристалл [Текст] / Н.Н Матвеев, Н.С. Камалова, Н.Ю. Евсикова // Пластические массы. - 2015. - № 3-4. - С. 30-32. 
5. Парфентьева, Н.А. О применении и решении задачи Стефана в строительной теплофизике [Текст] Н.А. Парфентьева, О.Д. Самарин, В.Л. Кашинцева // Вестник МГСУ, 2001. - № 4. - С. 323-328.

6. Парфентьев, Н.А. Математическое моделирование теплового режима конструкций при фазовых переходах [Текст] / Н.А. Парфентьев // Вестник МГСУ, 2011. - № 4. - С. 340-345.

7. Нагорнова, Т.А. Математическое моделирование процесса промерзания насыщенного влагой грунта [Текст] / Т.А. Нагорнов // Известия Томского политехнического университета. - 2005. - Т. 308. - № 6. - С. 127129.

8. Карслоу, Г. Теплопроводность твердых тел [Текст] / Г. Карслоу, Д. Егер. - М.: Наука, 1964. - 488 с.

9. Данилюк, И.И. О задаче Стефана [Текст] / И.И. Данилюк // Успехи математических наук. - 1985. - Т. 40. - № 5. - С. 133-185.

10. Мейрамов, А.М. Задача Стефана [Текст] / А.М. Мейрамов. - Новосибирск: Наука. Сибирское отделение, 1986. -239 с.

11. Красношлык, И.А. Численное решение задач с подвижными межфазными границами [Текст] / И.А. Красношлык, А.О. Богатырев // Вестник Черкасского университета, Серия «Прикладная математика. Информатика». - 2011. - Вып. 194. - С. 16-24.

12. Карташов, Э.М. Аналитическое решение однофазной задачи Стефана [Текст] / Э.М. Карташов, Г.С. Кротов // Математическое моделирование. - 2008, Т. 20. - № 3. - С.77-86.

13. Диткин, В.А. Интегральные преобразования и операционное исчисление [Текст] / В.А. Диткин, А.П. Прудников. - М.: Наука, 1961. -381 с.

14. Дмитриев, О.С. Прямая и обратная задачи теплопроводности в процессе прессования древесностружечных плит [Текст] / О.С. Дмитриев, С.В. Мищенко, А.Ю. Серегин // Вестник ТГТУ. - 2003. - Т. 9. - № 2. - C. 243-251.

15. Самарский, А.А. Вычислительная теплопередача [Текст] / А.А. Самарский, П.Н. Вабищевич. - М.: Едиторная УРСС, 2003. - $784 \mathrm{c}$.

16. Gupta, S.C. The classical Stefan Problem [Text] / S.C. Gupta // Basic Concepts, Modelling and Abalysis, Elsevier. $-2003 .-375 \mathrm{p}$.

17. Stefan, J. Ubereinige Probleme der Theorieder Warmeleitung [Text] / J. Stefan // Sitzungsberichte der Wissenschaften in Wien. MathematischNaturwissenschaftiescheKlasse. - 1889. Bd. XCVIII. Abth. - pp. 473-484.

18. Stefan, J Uber die Theorie der Eisbildung in Polarmeere Warmeleitung [Text] / J. Stefan // Sitzungsberichte der Keiserlicht Akademie der Wissenschaften in Wien. Mathematisch Naturwissenschaftiesche Klasse. - 1889. Bd. XCVIII. Abth. - pp. 965-983.

19. Лаврентьев, М.А. Методы теории функции комплексного переменного [Текст] / М.А. Лаврентьев, Б.В. Шабант. - М.: Наука, 1965.

20. Фихтенгольц, Г.М. Курс дифференциального и интегрального исчисления [Текст] / Г.М. Фихтенгольц. - М.: Физматлит, 2001.

21. Корн, Г. Справочник по математике для научных работников и инженеров [Текст] / Г. Корн, Т. Корн. M. 1968. $-720 \mathrm{c}$.

22. Zhou, Y Kinetic modelling of Diffusi-on-Controlled/Two -phase moving interacts problems [Text] /Y. Zhou, T.H. North // Modell. Simul. Mater. Sci. Eng. 1993. - Vol.1. - № 4. - pp. 505-516.

\section{References}

1. Chubinsky A.N., Sergeevichev V.V. Modelirovanie processov skleivanija drevesnyh materialov [Modeling the processes of gluing woody materials]. Saint Petersburg, 2007, 176 p. (In Russian).

2. Kumitsky B.M., Savrasova N.A., Chuikin S.V. Teplovye processy pri ostyvanii vodnogo bassejna [Thermal 
processes during cooling of an aquatic basin] Mezhdunarodnaja nauchno-prakticheskaja konferencija «Razvitie idej G.F. Morozova pri perehode k ustojchivomu lesoupravleniju» 20-21 aprelja 2017 g. [International Scientific and Practical Conference "Development of ideas G.F. Morozov in the transition to sustainable forest management "20 - 21 April 2017]. Voronezh, 2016, pp. 208-212. (In Russian).

3. Bartenev I.M., Vinokurov V.N. Jekologizacija tehnologii i lesnoj tehniki [Ecologization of technology and forest machinery [Text] Lesnoe hozjajstvo [Forestry]. 1992, no. 4-5, pp. 5-7. (In Russian).

4. Matveev N.N., Kamalova N.S., Evsikova N.Yu. Anomalii teplovyh svojstv celljulozy pri perehodah kristallkristall [Anomalies of the thermal properties of cellulose in crystal-crystal transitions] Plasticheskie massy [Plastic masses]. - 2015, no. 3-4, pp. 30-32. (In Russian).

5. Parfentieva N.A., Samarin O.D., Kashintseva V.L. O primenenii i reshenii zadachi Stefana v stroitel'noj teplofizike [On the application and solution of the Stefan problem in building thermophysics] Vestnik MGSU, 2001, no. 4, pp. 323-328. (In Russian).

6. Parfentiev N.A. Matematicheskoe modelirovanie teplovogo rezhima konstrukcij pri fazovyh perehodah [Mathematical modeling of the thermal regime of structures during phase transitions] Vestnik MGSU, 2011, no. 4, pp. 340345. (In Russian).

7. Nagornova T.A. Matematicheskoe modelirovanie processa promerzanija nasy-shhennogo vlagoj grunta [Mathematical modeling of the freezing process of a soil saturated with moisture] Izvestija Tomskogo politehnicheskogo universiteta [Izvestiya Tomsk Polytechnic University], 2005, Vol. 308, no. 6, pp. 127-129. (In Russian).

8. Karslou, G., Eger D. Teploprovodnost' tverdyh tel [Thermal conductivity of solids] Moscow, 1964, 488 p. (In Russian).

9. Daniljuk, I.I. O zadache Stefana [On the Stefan problem] Uspehi matematicheskih nauk [Uspekhi Matematicheskikh Nauk], 1985, Vol. 40, no. 5, p. 133-185. (In Russian).

10. Mejramov A.M. Zadacha Stefana [Zadacha Stefan] Novosibirsk, 1986, 239 p. (In Russian).

11. Krasnoshlyk I.A., Bogatyrev A.O. Chislennoe reshenie zadach s podvizhny-mi mezhfaznymi granicami $[\mathrm{Nu}-$ merical solution of problems with mobile interphase boundaries] Vestnik Cherkasskogo universiteta [Bulletin of the University of Cherkassy], 2011, Vol. 194, pp. 16-24. (In Russian).

12. Kartashov Je.M., Krotov G.S. Analiticheskoe reshenie odnofaznoj zadachi Stefana [Analytical solution of the single-phase Stefan problem] Matematicheskoe modelirovanie [Mathematical modeling], 2008, Vol. 20, no. 3, pp.7786. (In Russian).

13. Ditkin V.A., Prudnikov A.P. Integral'nye preobrazovanija i operacionnoe ischislenie [Integral transformations and operational calculus] Moscow, 1961, 381 p. (In Russian).

14. Dmitriev O.S., Mishchenko S.V., Seregin A.Yu. Prjamaja i obratnaja zadachi teploprovodnosti v processe pressovanija drevesnostruzhechnyh plit [Direct and inverse problems of heat conduction in the process of pressing wood chipboards] Vestnik TGTU [Bulletin of TSTU]. 2003, Vol. 9, no. 2, pp. 243-251. (In Russian).

15. Samarsky A.A., Vabishhevich P.N. Vychislitel'naja teploperedacha [Computational heat transfer] Moscow, 2003, 784 p. (In Russian).

16. Gupta S.C. The classical Stefan Problem. Basic Concepts, Modelling and Abalysis, Elsevier, 2003, 375 p.

17. Stefan J. Ubereinige Probleme der Theorieder Warmeleitung. Sitzungsberichte der Wissenschaften in Wien. MathematischNaturwissenschaftiescheKlasse. 1889. Bd. 98. Abth, pp. 473-484.

18. Stefan J Uber die Theorie der Eisbildung in Polarmeere Warmeleitung. Sitzungsberichte der Keiserlicht Akademie der Wissenschaften in Wien. Mathematisch Naturwissenschaftiesche Klasse, 1889. Bd. 98. Abth, pp. 965983.

19. Lavrentiev M.A., Shabant B.V. Metody teorii funkcii kompleksnogo peremennogo [Methods of the theory of a function of a complex variable] Moscow, 1965. (In Russian).

20. Fikhtengolts G.M. Kurs differencial'nogo i integral'nogo ischislenija [Course of differential and integral cal- 
culus] Moscow, 2001. (In Russian).

21. Korn G., Korn T. Spravochnik po matematike dlja nauchnyh rabotnikov i inzhenerov [Handbook on Mathematics for Scientists and Engineers]. Moscow, 1968, 720 p. (In Russian).

22. Zhou Y., North T.H. Kinetic modelling of Diffusi-on-Controlled/Two -phase moving interacts problems. Modell. Simul. Mater. Sci. Eng. 1993, Vol.1, no. 4, pp. 505-516.

\section{Сведения об авторах}

Кумицкий Борис Михайлович - доцент кафедры теплогазоснабжения и нефтегазового дела ФГБОУ ВО «Воронежский государственный технический университет», кандидат физико-математических наук, доцент, г. Воронеж, Российская Федерация; e-mail: boris-kum@mail.ru

Малюков Сергей Владимирович - доцент кафедры механизации лесного хозяйства и проектирования машин ФГБОУ ВО «Воронежский государственный лесотехнический университет имени Г.Ф. Морозова», кандидат технических наук, г. Воронеж, Российская Федерация; e-mail: maljukov-sergejj@rambler.ru

Саврасова Наталья Александровна - доцент кафедры физики и химии Военного учебно-научного центра Военно-воздушных сил «Военно-воздушная академия имени профессора Н.Е. Жуковского и Ю.А. Гагарина», кандидат физико-математических наук, г. Воронеж, Российская Федерация; e-mail: savrasova-nataly@mail.ru

Чуйкин Сергей Владимирович - доцент кафедры теплогазоснабжения и нефтегазового дела ФГБОУ ВО «Воронежский государственный технический университет», кандидат технических наук, г. Воронеж, Российская Федерация; e-mail: ser.chu@mail.ru

\section{Information about authors}

Kumitsky Boris Mikhailovich - Associate Professor of the Department of Heat and Gas Supply and Oil and Gas Business, Federal State Budget Education Institution of Higher Education Voronezh State Technical University, PhD in Physical and Mathematical Sciences, Associate Professor, Voronezh, Russian Federation; e-mail: boris-kum@mail.ru

Malyukov Sergey Vladimirovich - Associate Professor Department of Forestry Mechanization and Machine Design, Federal State Budget Education Institution of Higher Education «Voronezh State University of Forestry and Technologies named after G.F. Morozov», $\mathrm{PhD}$ in Engineering, Voronezh, Russian Federation; e-mail: maljukov-sergejj@rambler.ru.

Savrasova Natalia Aleksandrovna - Associate Professor of the Department of Physics and Chemistry of the Military Training and Research Center of the Air Force «The Air Force Academy named after Professor N.E. Zhukovsky and Yu.A. Gagarin», PhD in Physical and Mathematical Sciences, Voronezh, Russian Federation; e-mail: savrasova-nataly@mail.ru

Chuykin Sergey Vladimirovich - Associate Professor of the Department of Heat and Gas Supply and Oil and Gas Business, Federal State Budget Education Institution of Higher Education «Voronezh State Technical University», PhD in Engineering, Voronezh, Russian Federation; e-mail: ser.chu@mail.ru 